\title{
ENFOQUE SOCIOLÓGICO DE DIVERSAS CONCEPCIONES DE SALUD
}

\author{
Ricardo Moragas \\ (Universidad de Barcelona) \\ (Universidad de Rhode Island)
}

El bien salud puede ser observado desde diversas perspectivas para esclarecer su significado conservando siempre un enfoque sociológico. Este artículo examina el campo de la sociología de la salud, de la enfermedad, y de la medicina, pasando a continuación al análisis de las concepciones médicas de salud: las cuales se dividen en tres apartados: concepción somático-fisiológica basada en el examen del organismo físico; concepción psíquica la cual examina lo intangible del ser humano en salud y enfermedad, y concepción sanitaria que versa sobre la salud de las poblaciones. A continuación se detallan las concepciones de ciencias sociales: concepción político-legal de salud o derecho a la salud; concepción económica que analiza el precio de la salud y el coste de la enfermedad, y concepción social que proporciona perspectivas sobre relaciones sociales, roles y salud. El artículo finaliza con una concepción ideal de salud como estado inalcanzable, pero destacando las funciones positivas de dicha concepción. 

El objetivo del presente artículo es analizar con una perspectiva sociológica algunas de las concepciones fundamentales de la salud como bien del que todos los ciudadanos desean participar. Nuestro enfoque introductorio persigue, en un número monográfico como el presente dedicado a sociología y medicina, ilustrar la importancia de la salud como concepto clave y como bien deseado que rige los esfuerzos de los profesionales de la medicina y alumbra las esperanzas de todo ser humano. No hace falta justificar el presente enfoque sociológico, ya que la medicina posee una larga tradición la cual reconoce la importancia de los factores sociales en su ejercicio, pero sí vale la pena aclarar la selección del concepto de salud, clave de cualquier planteamiento sistemático de las relaciones entre medicina y sociedad.

\section{Sociología de la salud, de la enfermedad y de la medicina}

A nuestro juicio, el término sociología de la medicina es parcial y refleja tanto la importancia de esta profesión como la inferioridad de los sociólogos cuando se acercan al fenómeno de la salud y la enfermedad. Esta situación de inferioridad de la sociología respecto a la medicina se corresponde estrechamente con el desigual desarrollo de la teoría y práctica en ambas disciplinas. Mientras que muy pocas personas conocen los fundamentos de la sociología y (muy a pesar nuestro) para qué sirve un sociólogo, ninguna persona en una sociedad moderna ignora que existe una teoría médica y que la práctica de la medicina posee una utilidad inmediata. Una vez más recogemos en esta comparación el viejo tema del desigual desarrollo de las ciencias de la naturaleza (medicina) y de la sociedad (sociología). Se podrá objetar válidamente que la medicina no es puramente ciencia natural y que tiene en consideración los aspectos sociales del enfermo; pero la observación se vuelve a nuestro favor cuando comparamos los éxitos terapéuticos que consi- 
gue la medicina entre las enfermedades de naturaleza fundamentalmente orgánica ( $\mathrm{y}$ por tanto con terapéutica básicamente de ciencia natural) y las enfermedades de naturaleza psicosocial, enfermedades mentales. Sin negar los enormes adelantos que la medicina ha conseguido en la curación de las enfermedades mentales en las últimas décadas, debemos reconocer que los éxitos han sido mayores en la aplicación de fármacos, tranquilizantes y terapéutica orgánica que terapéutica psicosocial.

$\mathrm{Si}$ lo realmente importante para el hombre son la salud y la enfermedad como acontecimientos sociales, resulta sorprendente que a la sociología que analiza estos acontecimientos se la denomine «de la medicina» y no «de la salud» y «la enfermedad». Hay muchas razones que justifican la denominación sociología de la medicina y no podemos ignorarlas, comenzando por la mayor importancia de la profesión de medicina frente a la propia sociología. Razones de tipo histórico, las más válidas en el establecimiento de un campo, explican la temprana identificación de la sociología de la salud y de la enfermedad como sociología de la medicina. A partir de la segunda postguerra mundial cuando aparecen en Estados Unidos los primeros sociólogos enseñando e investigando sobre estas materias, lo hacen en Facultades de Medicina, siendo rápidamente identificados como siciólogos de la medicina, aunque la naturaleza de los temas que tratan es más amplia que lo que ese título parece indicar. Esta denominación es en el mejor caso parcial y no responde a los términos utilizados en la identificación de otros campos sociológicos. Por ejemplo, los sociólogos interesados en el fenómeno social de la religión no denominan a su disciplina sociología del sacerdocio, ya que sus intereses rebasan la actividad de los ministros del culto y se dirigen a toda la amplitud del hecho religioso. El intentar denominar a las materias que trata la sociología de la medicina, sociología de la salud, no es un mero capricho nominalista sino que se considera que el identificar adecuadamente el objeto de conocimiento ayuda a establecer las adecuadas relaciones entre profesionales y sobre todo a comunicar el mensaje con mayor exactitud al público. Sin embargo, si de salud se trata, es obvio que ello supone el tratamiento de la enfermedad y actividades y personas con la misma conectadas. Nuestra preferencia por el concepto de salud sobre el de enfermedad se basa en que es más positivo, pues destaca el bien físico, psíquico y social que todo el mundo desea alcanzar o conservar, según nos dice el organismo especializado de las Naciones Unidas al respecto y que se denomina precisamente «Organización Mundial de la Salud» y no «de la Enfermedad».

Debe indicarse no obstante que la mal llamada sociología de la medicina ha tratado otros muchos temas aparte de la profesión médica, de- 
dicando atención a las relaciones entre el sistema sanitario y el económico y social, a los roles que juegan el enfermo y otros profesionales no médicos en la salud, a las bases políticas de los sistemas sanitarios, a las organizaciones sanitarias como hospitales y departamentos sanitarios, etc. ${ }^{1}$ Este mismo número de «Papers: Revista de Sociología» recoge la tradición de identificar como sociología de la medicina un temario más extenso que el de la principal profesión responsable por la salud, $y$ así vemos que el contenido del mismo, aun dedicado en parte a la profesión médica, es más amplio que esta actividad profesional. Existe finalmente una razón sociológica, que no se debe olvidar para hablar de sociología de la salud y no meramente de sociología de la medicina, y es que una visión científica de nuestra disciplina, consagrada por muchos autores, utiliza el esquema institucional para tratar de las diversas actividades sociales que satisfacen necesidades permanentes en la sociedad, constituyendo esta serie de actividades instituciones sociales que originan a su vez campos especializados de la sociología. Así por ejemplo, la necesidad de procreación y educación de los hijos origina la institución familiar, la necesidad de distribución del poder la institución política dando lugar a las sociologías especiales de la familia y de la política. De la misma manera se puede ver que la necesidad social de asegurar el máximo bienestar físico, psíquico y social a la totalidad de los miembros de una sociedad origina la sociología especial de la salud. El denominar a este campo sociología de la medicina supone reducirlo a un enfoque parcial de sociología de las ocupaciones o de sociología del trabajo, que es a su vez otra institución social por derecho propio.

1. Para una buena visión de las aportaciones de la sociología a la salud y enfermedad ver P. Kendall y G. Reader «Contributions of Sociology to Medicine» en Freeman, Levine y Reeder (eds.), Handbook of Medical Sociology, 2 ed. «Englewood Cliffs: Prentice Hall», 1972) pp. 1-29. En los Estados Unidos ha predominado una clasificación que procede del artículo de R. Straus, "The Nature and Status of Medical Sociology», American Sociological Review, 22 (1957), pp. 200-204, en el cual se diferencia entre sociología en la medicina que correspondería al interés aplicado a un problema médico y sociología de la medicina que correspondería al interés en la salud y enfermedad como cualquier otra institución social. En la práctica la diferenciación es difícil de mantener tanto para los profesionales como para sus aportaciones. 


\section{La sociologia y las diversas concepciones de salud}

Sin intentar recabar para la sociología el antiguo ideal comtiano de la ciencia que engloba a las restantes en una cadena de complejidad y jerarquía crecientes, es evidente que la visión sociológica posee características de generalidad en el enfoque de los asuntos sociales. Al tratar de las concepciones de salud se pretende dar una visión general del punto de vista que diversas disciplinas utilizan. No se intenta llevar a cabo una taxonomía de los diversos enfoques bajo los que puede analizarse la salud, según las disciplinas diversas que se ocupan de la misma, sino ver la importancia social que determinada concepción de salud ha tenido en cierto momento histórico y cómo esta aportación ha contribuico a elaborar la visión global de lo que representa la salud en el mundo contemporáneo. Este enfoque no pretende por tanto ser especializado en cada apartado, ya que para ello habría que recurrir a los planteamientos estrictos de cada disciplina, sino sociológico, destacando lo que es general y social para cada materia.

Las diferentes concepciones que se detallan a continuación constituyen categorías interrelacionadas en muchos aspectos; no obstante la clasificación es necesaria para destacar los enfoques respectivos. Se han seleccionado siete concepciones de salud clasificadas en tres grupos: (A) concepciones médicas de salud, (B) concepciones de ciencias sociales y (C) concepción ideal de salud. Entre las concepciones médicas detallamos la somático-fisiológica, la psíquica y la sanitaria, entre las concepciones de otras disciplinas, la político-legal, la económica y la social, para finalizar con la concepción ideal de salud como categoría única que recoge anhelos y aspiraciones. Al final se recogen, en la Tabla 1, las diversas concepciones en un esquema breve de lo que suponen.

Las concepciones médicas de salud se basan en el punto de vista de la medicina como actividad profesional y que se ha dividido en tres apartados según sea el énfasis de la práctica médica, en la salud somático-fisiológica, en la psíquica o en la sanitaria.

1. Concepción somática-fisiológica. - Esta concepción parte de la realidad de la enfermedad en el organismo físico, la cuat ha sido la constante de mayor importancia en la historia de la humanidad y aún lo sigue siendo en la mayoría de los países. Salud es, en dicha concepción, bienestar del cuerpo y del organismo físico, y la enfermedad es el proceso que altera este bienestar. Esta concepción de salud destaca su carácter residual como estado de ausencia de enfermedad o de recuperación de la salud subsiguiente a un proceso morboso. Aunque actualmente ya 
no se considera dicha concepción válida, en especial en los países desarrollados, donde la prevalencia de las enfermedades somáticas puras es muy inferior al pasado, es evidente el peso que en la opinión colectiva y en la propia práctica médica tiene la concepción somático-fisiológica de salud. Esquemáticamente puede enunciarse esta concepción diciendo que si el organismo físico no posee alteraciones visibles existe salud, y sólo cuando baya una alteración del soma existirá enfermedad. En su versión extrema esta concepción ya no es válida, pues en todo tipo de culturas se admite la existencia de la enfermedad no somática; pero lo importante es lo que la práctica médica ha percibido como campo de acción propio, y es evidente que la mayor parte del desarrollo de la medicina viene de la mano de los adelantos en las ciencias naturales. Sólo la psiquiatría que tanto desarrollo ha tenido en los últimos años, pero aún situada a gran distancia de la medicina somática, se enfrenta a la concepción somática de salud. ${ }^{2}$

La conexión medicina-organismo físico enfermo es una constante histórica que se repite hasta nuestros días, y hace falta llegar al siglo pasado para que se impongan otras concepciones de la salud y del trabajo médico, como la medicina preventiva y social, la sanidad o la psiquiatría. Históricamente, la concepción somática de la salud se ha reflejado en la práctica médica que ha utilizado hasta hace poco fundamentalmente instrumentos fisicoquímicos para actuar en el organismo; es evidente que muchos médicos en su práctica diaria recurrían en la relación médicoenfermo al apoyo psicológico, al consejo familiar y a una terapéutica difusa que cada profesional elaboraba de acuerdo con su concepción individual de las necesidades del enfermo; no obstante, la enseñanza de la medicina, la exploración y examen del enfermo y la terapéutica habitual se basaban principalmente en la identificación de alteraciones en el organismo fisiológico. No en vano se conocía antiguamente a los médicos con el nombre de «físicos», ya que tanto por sus instrumentos como por sus actos la profesión se identificaba por el público con la manipulación de los elementos físicos del cuerpo humano. El éxito de la propia medicina somática contribuyó directamente a que ésta fuera la forma fundamental de hacer medicina, y a mediáa que los diferentes descubrimientos en biología, física, y química demostraban su gran eficacia para diagnosticar la enfermedad y curar el organismo físico, el

2. Para algunos enfoques de salud y enfermedad que reconocen to dificil de una definición estricta, ver R. Wilson, The Sociology of Healtb: An Introduction, cap. 1, «Defining Health and Illness», (Nueva York: Random House, 1970), pp. 3-12. 
«Papers»: Revista de Sociología

TABLA 1

Esquema de las concepciones de salud

\begin{tabular}{|c|c|c|c|c|}
\hline Concepción & $\begin{array}{c}\text { Nota carac- } \\
\text { teristica }\end{array}$ & Historia & Disciplina & $\begin{array}{l}\text { Métodos } \\
\text { trabajo }\end{array}$ \\
\hline $\begin{array}{l}1 \text { - SOMATICO } \\
\text { FISIOLOGICA }\end{array}$ & $\begin{array}{l}\text { Salud como au- } \\
\text { sencia de enfer- } \\
\text { medad. } \\
\text { Objetividad. }\end{array}$ & $\begin{array}{l}\text { Desde los orige- } \\
\text { nes a la presen- } \\
\text { te medicina. }\end{array}$ & Medicina clínica. & $\begin{array}{l}\text { Exploración del } \\
\text { cuerpo. Examen } \\
\text { de signos y sín- } \\
\text { tomas. Análisis } \\
\text { varios. }\end{array}$ \\
\hline 2 - PSIQUICA & $\begin{array}{l}\text { Salud de lo que } \\
\text { no es el cuerpo } \\
\text { tangible. Subjeti- } \\
\text { vidad. }\end{array}$ & $\begin{array}{lr}\text { Inicio s. } & \text { xumr. } \\
\text { Desarrollos } & \text { re- } \\
\text { cientes } & \text { desde } \\
\text { principios } & \text { s. } x x .\end{array}$ & $\begin{array}{l}\text { Psiquiatría. Psi- } \\
\text { cologia médica. }\end{array}$ & $\begin{array}{l}\text { Exploración psi- } \\
\text { quiátrica indivi- } \\
\text { dual. } \\
\text { Psicoanálisis. } \\
\text { Técnicas de gru- } \\
\text { po. }\end{array}$ \\
\hline 3 - SANITARIA & $\begin{array}{l}\text { Salud como es- } \\
\text { tado positivo y } \\
\text { colectivo. Trans- } \\
\text { misión social de } \\
\text { la enfermedad. } \\
\text { Prevención. }\end{array}$ & $\begin{array}{l}\text { Medidas profilác- } \\
\text { ticas desde la an- } \\
\text { tigüedad. Desa- } \\
\text { rrollo científico s. } \\
\text { xIx. }\end{array}$ & $\begin{array}{l}\text { Medicina preven- } \\
\text { tiva y social. } \\
\text { Sanidad y salud } \\
\text { rública. }\end{array}$ & $\begin{array}{l}\text { Encuesta sanita- } \\
\text { ria, } \\
\text { Epidemiologfa. } \\
\text { Educación sanita- } \\
\text { ria. }\end{array}$ \\
\hline $\begin{array}{l}4 \text { - POLITICO } \\
\text { LEGAL }\end{array}$ & $\begin{array}{l}\text { Salud como de- } \\
\text { recho y obliga- } \\
\text { ción universal. } \\
\text { Reconocimiento } \\
\text { legal y participa- } \\
\text { ción estatal. }\end{array}$ & $\begin{array}{l}\text { Desde las revo- } \\
\text { luciones políticas } \\
\text { a los sistemas de } \\
\text { seguros sociales y } \\
\text { de seguridad so- } \\
\text { cial. }\end{array}$ & $\begin{array}{l}\text { Derechos funda- } \\
\text { mentales. Dere- } \\
\text { cho de la seguri- } \\
\text { dad social a la } \\
\text { asistencia sanita- } \\
\text { ria. }\end{array}$ & $\begin{array}{l}\text { Leyes fundamen- } \\
\text { tales y constitu- } \\
\text { cionales. Leyes y } \\
\text { reglamentos de } \\
\text { la seguridad so- } \\
\text { cial. Programas } \\
\text { políticos. }\end{array}$ \\
\hline 5 - ECONO- & $\begin{array}{l}\text { Salud como con- } \\
\text { dicionante de la } \\
\text { produtcividad del } \\
\text { factor humano. } \\
\text { Precio de la sa- } \\
\text { lud y costo de la } \\
\text { enfermedad. }\end{array}$ & $\begin{array}{l}\text { Principios del s. } \\
\text { xx en países in- } \\
\text { dustrializados. }\end{array}$ & $\begin{array}{l}\text { Economía sanita- } \\
\text { ria y del trabajo. } \\
\text { Planes de desa- } \\
\text { rrollo de recur- } \\
\text { sos humanos. }\end{array}$ & $\begin{array}{l}\text { Análisis de cos- } \\
\text { tos de la enfer- } \\
\text { medad y de al- } \\
\text { ternativas para } \\
\text { las inversiones sa- } \\
\text { nitarias. }\end{array}$ \\
\hline $6-$ SOCIAL & $\begin{array}{l}\text { Salud como par- } \\
\text { ticipación social. } \\
\text { Relatividad cul- } \\
\text { tural de la salud } \\
\text { y la enfermedad. }\end{array}$ & $\begin{array}{l}\text { Posterior a la II } \\
\text { Guerta Mundial. }\end{array}$ & $\begin{array}{l}\text { Sociologia de la } \\
\text { salud, la enfer- } \\
\text { medad y la me- } \\
\text { dicina. }\end{array}$ & $\begin{array}{l}\text { Métodos de in- } \\
\text { vestigación social. } \\
\text { Análisis de toles } \\
\text { y de grupos. }\end{array}$ \\
\hline $7-$ IDEAL & $\begin{array}{l}\text { Salud como esta- } \\
\text { do ideal no al- } \\
\text { canzable. }\end{array}$ & $\begin{array}{l}\text { Desde la anti- } \\
\text { güedad clásica al } \\
\text { presente. }\end{array}$ & $\begin{array}{l}\text { Literatura. Antro- } \\
\text { pología. Otras } \\
\text { disciplinas. }\end{array}$ & $\begin{array}{l}\text { Elaboración mo- } \\
\text { delos de salud. }\end{array}$ \\
\hline
\end{tabular}

movimiento se aceleraba y generaba su propia demanda, demostrando la rentabilidad de las investigaciones en las ciencias naturales aplicadas al arte de curar. Por cualquier criterio que se examine hasta hace poco, la medicina ha sido fundamentalmente medicina somática, aunque algunos de sus profesionales incluyeran en su práctica un reconocimiento 
empírico de factores no somáticos en la génesis y terapéutica de la enfermedad. Tomemos como ejemplo los premios Nobel de Medicina concedidos desde 1901 hasta 1966; sólo en 1949 se otorgó a un descubrimiento que se refiere a la enfermedad mental, concedido a A. Egas Moniz, de Portugal, por su descubrimiento del valor terapéutico de la leucotomía en las psicosis, y aun en este caso se trata de una intervención quirúrgica y no de un tratamiento psicosocial, aunque la enfermedad sea mental.

La concepción somática de salud ha vivido durante la mayor parte de la historia más pendiente de la enfermedad que de la salud y es lógico que así fuera dada la magnitud del problema con que se enfrentaba. Es necesario llegar al siglo pasado para que la salud orgánica aparezca no sólo como liberación de la enfermedad sino como un estado positivo al que hay que acceder a través de prácticas preventivas. A partir de los grandes descubrimientos bacterológicos, del desarrollo de la inmunología, de la medicina preventiva, de la salud pública y otras disciplinas, se descubre, incluso al nivel puramente orgánico, que la salud es algo más complejo e importante que la mera ausencia de enfermedad o enfermedad compensada.

A pesar de las características objetivas que parece asumir el concepto de la salud orgánica, interesa a la sociología destacar que la percepción y vivencia de la salud y enfermedad no es uniforme para todos los individuos y que las diferencias se estructuran a lo largo de grupos sociales fácilmente identificables. El nivel socio-económico o educacional, la clase social, el grupo de ocio al que se pertenece, la religión, son todos ellos factores que influyen en la forma que los diferentes individuos viven y perciben la enfermedad somática; valga de ilustración la respuesta de un individuo de clase baja respecto a la enfermedad: «Algunas personas pueden meterse en cama debido a algo en casi cualquier momento, pero la mayor parte de nosotros no podemos enfermar incluso cuando lo necesitamos.» ${ }^{3}$

2. Concepción psíquica. - Lo psíquico se opone tradicionalmente a lo orgánico como lo subjetivo a lo objetivo, pero debido a la estrecha interrelación en el organismo humano entre cuerpo y psique, la experiencia de salud no puede restringirse a la mera salud orgánica. No obstante, frente a los logros e historia de la medicina que ha sido fundamentalmente hasta época reciente medicina somática, la salud psí-

3. Koos, Farl, The Health of Regionville (Nueva York: Columbia University Press, 1954) p. 30. 
quica aparece como una categoría residual o al menos de importancia menor en cantidad o intensidad. Lo psíquico era hasta hace poco lo no explicable orgánicamente, poseyendo el médico menos instrumentos de análisis, de diagnóstico y de terapéutica que en el caso de la medicina somática. A medida que se logran mayores niveles generales de salud física en sociedades desarrolladas, es cuando la medicina comienza a ser psicosomática científicamente, a reconocer la naturaleza sutil de las relaciones entre organismo físico y psiquismo y a incorporar los profesionales en su práctica principios y terapéutica científica de orden psíquico. ${ }^{+}$

Hasta que no se desarrollan las modernas teorías de la personalidad y aparece la psiquiatría científica para el tratamiento de los síndromes patógenos del psiquismo, no existe una teoría suficientemente sólida en la que basarse la medicina. Sin embargo, es necesario reconocer que, pesar de los recientes desarrollos, desde la revolución de Freud y los conductistas hasta las más modernas interpretaciones de la personalidad, existe un subdesarrollo de las teorías psíquicas. Sigue siendo aún cierto actualmente que se sabe mucho más sobre la base somático-fisiológica de la enfermedad que sobre la base psíquica de la misma, lo cual se debe en parte a la diferencia del objeto de conocimiento, en un caso el organismo fisiológico con límites ciertos y en el otro el psiquismo con toda su imprecisión de límites y posibilidades.

Ello no impide que en las modernas sociedades industriales se asista a un proceso de psicologización de muchas enfermedades, según afirman los médicos, en las cuales la componente fisiológica, aun existiendo, cede en importancia a los factores psíquicos. A veces la etiqueta dolencia psicosomática se aplica por los médicos a cuadros patógenos nuevos que a la luz de los actuales conocimientos de la medicina son de difícil interpretación. La limitación fundamental con que se enfrenta la concepción psíquica de salud es la subjetividad e indeterminación de sus manifestaciones, siendo el juicio profesional limitado, en comparación con la dolencia somática, ya que el enfermo puede siempre afirmar que no tiene salud, aunque objetivamente el médico no tenga base para aceptarlo. La sensación de bienestar psíquico es en última instancia una experiencia subjetiva frente a la que el médico no puede aducir pruebas objetivas de salud, de ausencia de gérmenes o causas patógenas, resultado de análisis y comprobaciones, como en el caso de las dolencias

4. Observaciones atinadas sobre la salud y enfermedad se encuentran en Fundación FOESSA «Informe sociológico sobre la situación social de España 1970» en el capítulo 13 «Sanidad» (Madrid: Euramérica, 1970). 
somáticas. El ejemplo más obvio del subjetivismo de la concepción psíquica de salud es el caso del dolor. Aun siendo aparentemente los tejidos musculares y nerviosos semejantes y la agresión patógena de la mis$\mathrm{ma}$ intensidad, la sensibilidad de dos individuos puede ser muy diversa, sin que puedan establecerse medidas científicas objetivas de la diversa intensidad del dolor que dos pacientes sufren.

Como veremos a continuación, aparte de la sensación diferencial individual debida a la naturaleza única de cada individuo, existe un condicionamiento social de la salud, que examinaremos más tarde, atribuible al hecho de que cada persona como ser social pertenece a un grupo social concreto y ha sido educada en el seno de una cultura específica que le ha preparado para percibir la salud y enfermedad en un determinado contexto.

Se ha mencionado antes que cualquir médico responsable intenta llegar a la experiencia subjetiva de la salud psíquica a través de la relación médico-enfermo, la cual desde la medicina clásica, pasando por la humanística, hasta la moderna, se ha considerado fundamentalmente un proceso único no sometible a normas generales y que debía elaboratse a la luz de cada caso concreto. Esta relación, manifestada fundamentalmente a través del diálogo, reconoce la individualidad de cada enfermo e intenta llegar a conclusiones prácticas para la mejor terapéutica en cada caso; pero como todo médico experimentado reconoce, los principios en que se basa esta relación son mucho menos científicos que los que puede aplicar en el examen y terapéutica de la componente somática del enfermo. El médico se limita en muchos casos a reconocer la necesidad de enfrentarse con la componente psíquica de la salud del enfermo y sabe que su consejo y presencia curan, pero las formas en que lo consigue y una explicación sistemática de su acción no han podido nunca establecerse con la claridad del diagnóstico y terapéutica somática. ${ }^{5}$

Un aspecto que en los últimos tiempos ha tenido reconocimiento explícito por la psicología médica, ha sido la variabilidad de la personalidad del enfermo durante las diversas etapas del proceso patógeno, de tal forma que su concepción de salud psíquica va evolucionando en el curso de la dolencia. La entrada de las ciencias sociales en el campo de

5. Existe una extensa tradición en medicina sobre la relación médico-enfermo por parte de todas las escuelas que, aunque partiendo de presupuestos diferentes, reconoce la sutileza e importancia de esta relación para la salud global. Entre nosotros debe destacarse la aportación de Laín Entralgo a esta relación desde el punto de vista de la medicina antropológico-humanística. 
Ia salud y de la enfermedad ha ayudado a estos exámenes y hoy se analizan las etapas de la enfermedad y su influencia sobre el psiquismo del enfermo, la secuencia temporal de la relación con el médico y sus auxiliares, la evolución longitudinal de la enfermedad, etc., destacando en estos análisis la plasticidad de las reacciones del ser enfermo y las profundas variaciones que la enfermedad ejerce sobre su psiquismo.

Finalmente hay que mencionar en la concepción psíquica de salud aquellos casos en que la enfermedad es fundamentalmente del psiquismo y por tanto la disciplina apropiada para tratarla es la psiquiatría. El desarrollo de la psiquiatría científica es relativamente reciente y sus logros notables, algunos de ellos basados precisamente en el control de las alteraciones somáticas del paciente, lo cual posibilita un tratamiento a través de técnicas psicosociales más adecuado. Una vez más se demuestra la estrecha interrelación entre las diversas concepciones de salud. Lo que intenta ser una presentación sistemática no debe confundirse con la riqueza de los enfoques en la vida real: una vez más debemos repetir que las concepciones de salud se dan entremezcladas en la realidad en especial en estas tres primeras concepciones médicas: somática, psíquica y sanitaria.

3. Concepción sanitaria. - La concepción sanitaria es concepción médica de salud, pues tanto sus profesionales como los objetivos que persiguen se dirigen al mantenimiento, preservación o recuperación de la salud, pero su énfasis no radica en la salud individual, fundamental en las anteriores concepciones de salud, sino en la salud colectiva de una población o comunidad. Esta consideración colectiva de la salud como fenómeno objeto de la sanidad o salud pública la sitúa en estrecha relación con la sociología con la que comparte muchos aspectos, aunque sus objetivos sean diferentes. (Aquí se toman como equivalentes las materias de sanidad y de salud pública ya que, aunque existen ciertas diferencias según los autores y las latitudes, se las puede considerar disciplinas semejantes.) Siguiendo a Suchman, las semejanzas entre la sociología y la sanidad son su preocupación por las poblaciones, la orientación teórica hacia generalizaciones abstractas y un enfoque metodológico que destaca los métodos cuantitativos y estadísticos, poseyendo ambas

6. El tratamiento más sistemático de las etapas de la enfermedad sigue siendo el de E. Suchman, «Stages of Illness and Medical Care» en Journal of Health and Human Bebavior, 6 (1965): 114-128.

7. E. Suchman, Sociology and the Field of Public Health (Nueva York: Rusell Sage Foundation, 1963) p. 28. 
materias un interés semejante que se comprueba históricamente por el origen común en el área de la reforma social. La confluencia de la sociología y la sanidad se manifiesta en la creciente superposición, según dicho autor, en problemas sociales y sanitarios tales como el control de la población, la ancianidad, la delincuencia juvenil y la deficiencia mental. Los puntos comunes se mantienen en la consideración de la demografía y la ecología, en el énfasis en la predicción, en el punto de vista macroscópico, en los métodos epidemiológico y de encuesta social, con semejanza de técnicas, diseño de instrumentos y problemas de análisis.

La concepción sanitaria de salud se caracteriza por un enfoque positivo de los problemas destacando la naturaleza preventiva de los mismos: al no interesarse directamente por un caso concreto sino por agregados o grupos sociales le lleva a utilizar la estadística y dirigir sus conclusiones a niveles generales. Estas generalizaciones, a semejanza del diagnóstico individual de la medicina clínica, deben identificar el grado de salud o de enfermedad de una comunidad; para llegar a estas conclusiones la sanidad establece medidas de lo que se considera saludable para cada comunidad o región del país. Estas valoraciones de lo que se considera normal o patógeno no son uniformes, dentro de ciertos límites, pues las definiciones pueden oscilar entre márgenes. Para un país en vías de desarrollo las valoraciones que el agente sanitario deba aceptar como normales serán, aun a pesar suyo, muy diversas de las que aceptaría en un país industrializado. La normalidad se define en cada sociedad de acuerdo con un cúmulo de circunstancias económicas, sociales, del medio ambiente, climáticas, que condicionan lo que se considera aceptable como «normal» para cada sociedad. La idea de normalidad en salud no es ciertamente ajena a la práctica de la medicina, ya que el cínico utiliza constantemente en sus exámenes el concepto de norma o valor aceptable para medir la salud fisiológica, comparando las mediciones de un paciente individual con las habituales para los pacientes de la misma edad, sexo, ocupación, etc. Una desviación de la norma, en individuos como en grupos, no supone necesariamente un estado patógeno, pero indica al médico clínico o sanitario que existen unos factores a los que debe prestar especial atención.

Otra nota peculiar de la concepción sanitaria de la salud es el enfoque preventivo, más intenso que en las concepciones somática y psíquica. El técnico sanitario conoce por experiencia la importancia de las medidas preventivas $y$, lo que es más importante, el contenido de su trabajo se ha diseñado para evitar la enfermedad con la acción previa que alcance a toda la población potencial; el médico somatólogo o psi- 
quiatra puede tener la misma actitud preventiva, pero su contacto con el caso individual tiene lugar en la mayor parte de las ocasiones cuando ya se ha producido la agresión patógena, y su cometido es entonces fundamentalmente diagnóstico y terapéutico: ello se debe, aparte de su posible sensibilidad por un enfoque preventivo, al origen de su intervención que se reclama casi siempre cuando la enfermedad es real y no potencial, pues dadas las actitudes del público la medicina individual es aún fundamentalmente curativa y no preventiva. La concepción sanitaria, al no depender para su intervención de la demanda de servicios por la parte afectada, actúa con mayor independencia pudiendo realizar una labor preventiva imposible en la medicina individual contemporánea.

La concepción sanitaria de salud no aparece hasta el siglo pasado, cuando el propio desarrollo de la medicina somática y los progresos en política social y económica permiten que los respectivos Estados se planteen los problemas de salud como una responsabilidad pública. El enfoque global de estos problemas lleva rápidamente a la racionalidad de la prevención, la utilidad de las medidas de inmunización y la rentabilidad de los instrumentos preventivos. La magnitud de los problemas planteados por la industrialización y urbanización con el traslado de grandes masas humanas que pasan a vivir en condiciones infrahumanas en los cinturones industriales de las primeras ciudades fabriles acelera la necesidad de que el estado adopte una actitud decidida para tutelar la salud de la población; por otra parte, los grandes descubrimientos bacteriológicos facilitan la aplicación de medidas masivas a toda la población que confirman la eficacia de la acción preventiva.

La concepción sanitaria de la salud posee un contenido amplio que puede dividirse esquemáticamente en medio ambiente material y personas que lo ocupan; al tratar del medio ambiente físico se interesará por la totalidad del mismo: tierra, agua, aire y alimentos en sus diversas formas constituirán su objeto, analizando las condiciones que facilitan u obstaculizan la difusión de la enfermedad; al referirse a las personas, las agrupará por sus condiciones personales, edad, sexo, estado civil y su adscripción a determinados grupos sociales de residencia, trabajo, ocio, buscando regularidades en estas agrupaciones que permitan el establecimiento de programas para actuar sobre los mismos.

Finalmente, la gama de actividades de la sanidad hacen que su concepción sea más amplia que las concepciones somática y psíquica vistas; mientras éstas perseguían en la mayor parte de los casos la curación y en el mejor caso cierta información y prevención, la concepción sanitaria analiza situaciones, establece planes de acción, preventivos y curativos, y de control permanente, diseña programas de información y educa- 
ción para el público y de formación para el personal sanitario, organiza y coordina las actividades de los profesionales y de otras entidades y también investiga para mejorar la calidad de la acción sanitaria en todas las áreas que cubre. Esta variada gama de actividades excluye la realización aislada y privada de las mismas y ello desemboca en que la concepción sanitaria de salud es fundamentalmente una concepción médica, pero pública y estatal.

4. Concepción político-legal. - Con esta concepción de salud se dejan los enfoques fundamentalmente médicos y nos introducimos en las concepciones de ciencias sociales, reconocimiento de que la salud atrae por su importancia actual a otras disciplinas aparte de la medicina. La concepción político-legal de salud parte de la consideración de la misma como un bien general que a todos interesa; cuando este bien es tutelado por el ordenamiento jurídico de un país se convierte en derecho exigible por los ciudadanos según una amplia gama de variedades de reconocimiento. La tutela legal no llega procedente de la voluntad unilateral del Estado, sino que se origina debido a la valoración social del bien salud, que lleva a los ciudadanos a solicitar su protección jurídica. La trayectoria de este derecho se inicia como declaración programática en la constitución del estado para luego hacerse efectivo sobre determinadas categorías de personas, en el inicio normalmente a los trabajadores a través de sistemas de seguros sociales e irse extendiendo sucesivamente a otras categorías de personas, familiares de los anteriores, grupos especiales, hasta llegar a las formulaciones generales de la seguridad social comprensiva de la que no se excluye prácticamente a ningún ciudadano. Esta evolución es la típica seguida por los países capitalistas cccidentales, ya que en los países socialistas, la salud junto con la educación es una de las primeras preocupaciones del Estado y el establecimiento de un programa de asistencia sanitaria estatal general para toda la población, uno de los primeros actos de gobierno.

Históricamente, descle Bismarck, primer iniciador de un sistema de seguridad social, hasta Beveridge en el mundo capitalista occidental y en la Unión Soviética así como en las repúblicas socialistas del Este de Europa, el hecho fundamertal es la intervención progresiva e ineludible del Estado en la protección de la salud. Esta intervención decidida no se produce hasta que existe una conciencia social por parte de los ciudadanos que reclama una acción en este terreno por ser la salud un patrimonio colectivo importante. Para llegar a este momento han hecho falta las revoluciones políticas las cuales han proclamado los derechos fundamentales de la persona y del ciudadano, entre los que está el de- 
recho a la integridad física. Jurídicamente, más que de un derecho a la salud habría que hablar de un derecho a la integridad física, lo cual supone la protección por parte del Estado en aquellos casos en que la in. tegridad pueda verse en peligro. A este derecho a la integridad física corresponde correlativamente el deber y obligación de mantenerla reconocida por todos los códigos penales, que identifican los actos que atentan contra la misma como delitos. $\mathrm{El}$ atentar contra salud, propia o ajena, es penado por el Estado, pues supone un ataque a un bien común que debe protegerse.

El derecho y obligación a la salud se materializan en una gama de servicios amplios que se inician históricamente con el derecho a la asistencia médica, prosiguen con la asistencia para los fármacos, prótesis, tratamientos especializados, rehabilitación ${ }^{8}$ y prevención. El reconocimiento a los ciudadanos de los mencionados derechos no se hace normalmente en los países capitalistas de una vez sino que es progresivo, constituyendo cada etapa un episodio en la lucha por las conquistas sociales y políticas.'

La percepción de la salud en los modernos Estados occidentales como un bien real al que cada día es más difícil acceder con los medios privados, dado el coste de la moderna medicina, ha motivado que se acelerara la intervención del Estado para satisfacer esta necesidad, haciendo cada vez más público el tema de la salud y los medios para mantenerla y recuperarla. Los partidos políticos no han sido ajenos a este proceso y la salud se ha convertido en un arma política, pues al quedar siempre al margen de la cobertura ciertos servicios y determinadas categorías de personas, los programas que buscaban su protección encontraban un eco fácil, sin importar demasiado su filiación ideológica; con pocas excepciones los partidos han defendido la protección de la población, aunque las modalidades de cómo conseguirla hayan variado entre los mismos.

La concepción político-legal de salud parte pues del principio de la salud como derecho para toda la población con la correspondiente obligación de mantenerla, existiendo una serie de disposiciones legales que

8. Ver a este respecto R. Moragas, Rebabilitación: Un enfoque integral, cap. 8, «Derecho y Rehabilitación», que trata un aspecto del derecho a la salud (Barcelona: Vicens Vives, 1972).

9. Ejemplo típico de esta progresiva extensión de los beneficios de la asistencia sanitaria para hacer efectivo el derecho a la salud lo tenemos en España donde la Seguridad Social que comenzó como laboral ha ido extendiendo progresivamente su campo de acción a otras categorías de personas y sigue haciéndolo constantemente. 
regulan el derecho y una valoración social del mismo que le llevan a ser objeto de discusión e incorporación a programas políticos. Esta concepción se extiende a todas las latitudes y sistemas políticos $\mathrm{y}$, aunque las variedades de su reconocimiento como derecho sean diferentes entre diversos sistemas, existe un fondo común universal en la concepción político-legal de salud y éste es la valoración que de la misma han realizado las personas de cualquier credo o nacionalidad y que reconocen, junto con la declaración universal de los derechos humanos, la preeminencia de este derecho para todo ser humano en el mundo contemporáneo.

5. Concepción económica. - Esta concepción se impone en el mundo desarrollado debido a la importancia que la salud posee para la eficacia del factor humano en los procesos productivos a los que aporta un elemento único y costoso. En otras épocas históricas el factor humano no era un factor estratégico, dada su abundancia, lo cual llevaba a su poca estimación económica, mientras que un factor escaso como el capital atraía la atención principal del empresario. La situación actual se ha invertido totalmente y el factor humano constituye un elemento estratégico de los procesos productivos, dadas su escasez y exigencias de calidad, actuando en este caso la universal ley económica de que los recursos escasos tienen un mayor valor en el sistema económico, y de ahí la importancia en mantener la productividad humana con una buena salud.

Siempre se ha considerado que los recursos humanos formaban parte de la riqueza de un país, y los monarcas de la antigüedad, como los Estados del renacimiento, medían sus fuerzas respectivas por el número de sus súbditos capaces de empuñar las armas; ésta no era una mera valoración numérica del potencial humano pues había que reunir determinadas condiciones cualitativas de aptitud física para poder figurar en el ejército, entre las que figuraba el disfrutar de buena salud. Lo realmente nuevo hoy es el papel estratégico que económicamente el elemento humano juega en los procesos productivos siendo ello resultado de una lenta evolución social que ha alcanzado en el mundo contemporáneo sus niveles más elevados. El factor humano es valioso económicamente pues su remuneración constituye un apartado importante, el más importante en muchas actividades como las sanitarias, pero esta remuneración no es sólo fruto de unas leyes económicas que determinan el precio de los factores de la producción, sino resultado de un progreso económico conseguido por diferentes medios, entre ellos la lucha sindical y la política protectora del Estado para la fuerza de trabajo. 
La salud de la mano de obra de un país determinará directamente su productividad y es por ello que los gobiernos nacionales están interesados, a través de diversos sistemas, en mantener el mayor nivel de salud para sus trabajadores. La seguridad social comprensiva es la forma habitual de proteger al trabajador y conseguir una garantía de que la mano de obra poseerá un cierto nivel de salud, asegurando así la productividad del sistema económico. Las prestaciones sanitarias tienen actualmente en los sistemas de seguridad social una gran importancia económica, pues dado el elevado costo de la moderna medicina, permiten al beneticiario disfrutar de una asistencia a la que en muchos casos individualmente no tendría acceso. El derecho a la salud ha sido percibido rápidamente por el pueblo como uno de los derechos fundamentales que debería tener un reconocimiento inmediato, su realización se ha visto facilitada por la componente económica que informa a la salud y que, si interesaba a los trabajadores, también era deseada por las clases directivas, empresarios, técnicos en la empresa pública y privada, que veían en la mejor protección de la salud una garantía de mayor productividad y eficiencia del sistema económico. A esta componente económica de la salud se deben en parte los grandes progresos realizados en un espacio de tiempo relativamente reducido en la consecución del derecho a la salud.

La importancia de la concepción económica de la salud ha sido expuesta analíticamente por los economistas, los cuales han calculado para la totalidad de una población los costes de la enfermedad en la reducción de la capacidad productiva y el despilfarro económico que ello supone; sus investigaciones han quedado estereotipadas en la expresión que se ha hecho popular «el precio de la salud y el coste de la enfermedad»." Esta frase refleja el sencillo hecho de que para conseguir un adecuado estado de salud en una población es más económico invertir en actividades preventivas que esperar a que la enfermedad aparezca y tener que emprender la acción curativa.

En la consideración económica de la salud merece destacarse la importancia de los costes por mantenerla comparados con otros costes de

10. En nucstro país los planteamientos económicos de la salud son reducidos; la frase mencionada figura como apartado en un capítulo de la obra Introduccion a la Medicina Preventiva y social de E. Zapatero y F. Yuste (Barcelona: Pulso Editorial, 1971), cap. IV, tomada de Winslow. Los Planes de Desarrollo han dedicado cierta atención a la salud como factor en la adecuada utilización de los recursos humanos, pero en general no existen economistas interesados en estos temas y la carencia de un departamento ministerial responsable total para la sanidad dificulta un enfoque global de la economía de la salud en España. 
la vida contemporánea. Los gastos sanitarios globales y el coste de tratamientos específicos han aumentado en los últimos años a un ritmo más rápido que el coste general de la vida, lo cual se debe a diversas causas entre las que figura el mayor coste del personal sanitario y tecnología avanzada que se materializa en fármacos y equipo sanitario complejo. Dado que el costo sanitario es aportado en gran parte por el Estado, es fácil inferir las consecuencias políticas de unos costes crecientes frente a la opinión pública. La salud se hace cada día más costosa y las decisiones sobre cómo mantenerla y promoverla escapan al puro campo médico y aparecen profesionales de otras disciplinas entre los que se encuentra en lugar destacado el economista. Las decisiones en última instancia ya no son meramente técnicas del médico o del economista, sino que entran en el terreno de los valores más fundamentales en que se asienta la sociedad y son los políticos los que deben tomarlas, ya que la salud es un estado siempre mejorable y en el que las inversiones nunca son suficientes, dada la limitación de los recursos económicos y el carácter expansivo de las necesidades de salud.

Un ejemplo del tipo de decisiones que no se dejan solamente a los técnicos en ningún sistema sanitario, sean médicos o economistas, es el de las alternativas a las cuales deben aplicarse los recursos, ya que ello constituye una decisión eminentemente política. ¿Quién debe decidir, con un presupuesto limitado, si parte del mismo se aplica a prolongar la vida de los pacientes seniles o a detectar las anomalías congénitas en los recién nacidos? Ambas actividades deben realizarse y de hecho se llevan a cabo con diversos grados de intensidad en todas las naciones, pero la decisión crítica es hasta qué punto deben concentrarse los recursos en una actividad o en otra o repartirse entre ambas, ya que no se puede hacer todo al máximo y al mismo tiempo. En los sistemas occidentales que no poseen un sistema sanitario integral, las decisiones son el resultado de una negociación y del conflicto de intereses de los grupos partícipes en el sistema político. La importancia económica de la sanidad ha motivado que los políticos se interesen por la misma, aparte de su teconocimiento, por el volumen de la inversión, por su importan. cia para la productividad y por el impacto social que posee la necesidad de salud en la población.

6. Concepción social. - El que la salud ha superado el estricto cauce individual, para convertirse en un asunto social, es reconocido actualmente por la mayotía de los profesionales y del público. Salud y enfermedad no son acontecimientos individuales, sino sociales, y aunque se intentara tratarlos como hechos individuales, el hombre al ser miembro 
de una sociedad de la que deriva en buena parte su personalidad y estilo de vida, se convertirían en sociales. Para ser una persona sana, el hombre necesita de relaciones sociales con ciertas características y, cuando pierde la salud, aunque la causa sea fundamentalmente fisiológica, su curación se acelera si mantiene unos contactos sociales adecuados. El hombre enfermo o sano necesita el apoyo de los demás seres que se relacionan normalmente con él como representantes de diversos grupos con sus valores, objetivos y formas de comportamiento específicos.

Las relaciones sociales pueden ser causa coadyuvante de la salud o enfermedad, como sucede en el caso de las enfermedades fundamentalmente fisiológicas, o ser la sociabilidad la propia causa de falta de salud; en este último caso nos encontramos con un campo en continua expansión que es el de las enfermedades mentales. Estas enfermedades vienen constituidas por aquellas condiciones en las que lo patógeno es precisamente la rotura o inexistencia de unas relaciones sociales adecuadas. El término sociogénesis cubre una amplia gama de síndromes, la mayoría de los cuales tienen de común la falta de una etiología orgánica física. La falta o deficiencia de las relaciones sociales puede ser causa de enfermedad $\mathrm{y}$, aunque los niveles de diagnóstico y terapéutica sean inferiores en efectividad a las dolencias fisiológicas, la evidencia de las enfermedades mentales de la sociabilidad es un hecho empírico.

Aparte de ser las relaciones sociales causa directa de enferme$\mathrm{dad}$, como acabamos de indicar, también son importantes estas relaciones como coadyuvantes a la salud en procesos fundamentalmente somáticos, aunque como ya se ha indicado repetidamente no existen dolencias puramente de uno u otro tipo, pues en la realidad la psique siempre se mezcla con lo orgánico. En el caso de dolencias básicamente somáticas su identificación, diagnóstico, eficacia de la terapéutica, queda condicionada por la calidad de las relaciones sociales manifestadas a través de los grupos con los que se halla en contacto el sujeto enfermo; según sea el apoyo u oposición que dichos grupos exhiban frente a la enfermedad del sujeto, las reacciones del mismo, tanto somáticas como psíquicas, se verán afectadas. El rol social, o papel que cada individuo desempeña en la sociedad, no se representa aisladamente del resto de los seres con los que entra en relación, sino que viene condicionado por sus actitudes, influencias, comportamientos, existiendo un rol de persona sana y un rol de persona enferma, con los correspondientes derechos y deberes sociales asignados a los mismos, como ha señalado claramente Parsons."

La definición social de la salud y de la enfermedad consiste en que

11. The Social System (Nueva York: Free Press, 1951), cap. 10. 
cada sociedad evalúa to que es estar sano y enfermo, a la luz de una situación de hecho existente en la misma. La salud queda condicionada en cierta manera por una situación global de desarrollo económico, educativo, político, peculiar a cada país en un momento histórico determinado. Lo que hoy es salud para una sociedad, puede no serlo dentro de unos años, ya que la definición de salud es dinámica como otras concepciones sociales, y lo que es salud para una sociedad hoy, puede ser enfermedad para otra, por ser su definición más estricta. Por ejemplo, un nivel de consumo de calorías inferior a la media se considera en la mayoría de los países occidentales como un estado de falta de salud, mientras que el mismo consumo occidental de calorías en el sudeste asiático puede suponer un nivel superior al normal y una salud resultante superior. La salud es por tanto relativa al marco cultural en el cue se analiza, ya que no constituye solamente un estado objetivo de bienestar, sino una experiencia subjetiva valorada de acuerdo con los criterios sociales de lo que es prevalente en cada sociedad.

La salud como estado de aptitud para el desempeño de roles sociales se define tan variadamente como numerosos son los roles en la sociedad; tomando como ejemplo el rol laboral que puede desglosarse en multitud de puestos de trabajo, veremos que una misma condición afectará diferencialmente a dos sujetos en su rol, según sea el contenido de sus funciones. La paralización de una extremidad inferior es causa de falta de salud para el desempeño del rol de trabajador de la construcción, mientras que no invalida al directivo o técnico en el desempeño regular de sus funciones que no requieren desplazamientos.

Todo lo mencionado respecto a definición social de la salud según el estado de desarrollo del país de que se trate y según cuales sean los roles sociales que la persona deba cubrir nos lleva a enunciar lo que se ha denominado en breve expresión «relatividad cultural de la salud y la enfermedad»; ${ }^{12}$ ello quiere decir que cada grupo social, sea tan extenso como una nación o tan reducido como un grupo familiar, poseerá una definición peculiar de salud establecida de acuerdo con lo que se considera «normal» en dicho grupo y que esta definición influencia activamente la forma de sentirse sano o enfermo de las personas pertenecientes al mismo. La medida en que dicha influencia social actúa sobre el individuo para definir el estado de salud o enfermedad es una resultante de la personalidad de cada sujeto y del efecto que las presiones

12. La frase es utilizada por Parsons en un sentido más general en «Patients, Physicians and Illness», E. G. Jaco (ed.), Definitions of Health and Illness in the Light of Anzerican Values and Social Structure (Nueva York: Free Press, 1972), 2." ed., pp. 110-111 
del grupo producen en su comportamiento social. Lo único que puede afirmarse, a nivel general, es que existe esta influencia social y que en cada caso habrá que valorarla según las dos componentes del esquema, la presión del grupo y la personalidad del sujeto.

Aparte de las manifestaciones de la salud y enfermedad sobre la personalidad y roles sociales del individuo, debe señalarse un aspecto también social y que identifica los planteamientos contemporáneos de la salud; se trata del impacto social que posee actualmente la salud y la enfermedad al considerarse temas de interés no sólo para el individuo sino para toda la sociedad. Las preocupaciones por mantener niveles elevados de salud se manifiestan por el Estado con su perspectiva de eficaz utilización de los recursos económicos como ya se ha indicado en la concepción económica de salud, pero también es socialmente importante la cobertura del mayor número de personas con sistemas de seguridad social que incluya la asistencia sanitaria, la planificación de las actividades sanitarias, la discusión de los objetivos sanitarios con participación de los grupos afectados, terapeutas y pacientes que persiguen fines no siempre coincidentes. La sociología como ciencia de las relaciones sociales toma conciencia de todos los procesos sociales que aparecen y se desarrollan en torno a la salud analizándolos con sus instrumentos de investigación; como se ha indicado anteriormente, será difícil en algunas de sus contribuciones diferenciar lo que es investigación sanitaria de investigación sociológica, ya que ambas disciplinas tienen puntos de vista comunes en su consideración social de la salud y de los medios para alcanzarla y mantenerla. Con la concepción social de salud se finaliza la visión que las diferentes disciplinas desde las médicas a las de ciencia social tienen de la salud, se tratará finalmente de una concepción que aunque no atribuible a ninguna materia en concreto tiene importancia para todos.

7. Concepción ideal de salud. - Esta concepción supone un estado de salud no alcanzable por el hombre contemporáneo en su realidad diaria; sin embargo tiene importancia, pues las concepciones ideales son guías inspiradoras de los actos cotidianos, aunque se sepa que nunca van a alcanzarse plenamente dichos objetivos. La concepción ideal de salud más conocida es la propia definición que nos da de ella la Organización Mundial de la Salud, «estado de bienestar físico, mental y social completo y no meramente la ausencia de enfermedad o disminución»; esta definición es evidentemente ideal, pues dicho estado de salud es imposible conseguirlo para toda una población; sin embargo es una definición útil, pues sirve de guía para las actividades sanitarias que versan 
siempre sobre estados parciales de salud física, psíquica o social. Algunos dirán que tal definición es utópica y por tanto no sirve a los objetivos de las ciencias interesadas en la salud, pero ello no es cierto pues, como la sociología ha analizado frecuentemente, las utopías tienen importantes funciones sociales que cumplir cuando no constituyen una evasión de la realidad sino un mecanismo de motivación para formas sociales inadecuadas y que hay que reformar y mejorar.

Algunas personas consideran la concepción ideal de salud como la utopía de un individuo disfrutando del bienestar físico, psíquico y social en plentiud; y para siempre. Esta visión sería irreal por estática ya que ignoraría una componente fundamental de los procesos vitales cual es su dinamismo. La salud como fenómeno vital no puede ser un estado fijo que se alcance definitivamente gracias a la supresión de los agentes patógenos, pues ello supondría suprimir algo tan inherente al hombre como su evolución y permanente posibilidad de cambio físico, psíquico y social. La salud perfecta como la política sanitaria perfecta no se alcanzará nunca, como afirma Dubos; ello sería quizás posible para una colonia de hormigas o abejas con hábitos sociales fijos e instintivos, pero mientras los hombres no se conviertan en autómatas no existe posibilidad de proporcionarles una salud fija y estable para toda su vida. ${ }^{13}$

Es evidente que de la concepción ideal de salud hay que pasar en la práctica médica y sanitaria a niveles de salud aceptables o inaceptables y que vendrán condicionados por la situación global de cada sociedad, como se ha indicado anteriormente. Estos niveles serán siempre relativos a una circunstancia económica, política y científica concreta del momento histórico de aquella sociedad; la concepción ideal de salud servirá de fuerza motivante para mejorar los niveles de salud existente, aunque nunca se vaya a alcanzar el estado ideal de salud carente de toda enfermedad.

Las visiones de un estado ideal de salud de cierta literatura antropológica y social destacan la bondad del estado natural del ser humano, no pervertido por la civilización occidental; existe una amplia tradición en el pensamiento occidental en esta dirección, la cual va desde el salvaje natural de Rousseau hasta las descripciones idílicas de las sociedades primitivas por los antropólogos. Este punto de vista considera que la civilización contemporánea es la causa de todos los males que afligen al hombre y que, para librarse de ellos, la única solución es un retorno a estados de vida más primitivos, en los que se suprimirán las causas de nuestros problemas actuales. La ingenuidad del razonamiento no deja de reclutar adeptos continuamente, debido a que existe cierta verdad

13. Mirage of Health (Nueva York, Doubleday, 1959), p. 227. 
parcial en este tipo de afirmación. Nuestra forma de vida contemporánea es responsable de varios tipos de enfermedades y carencias de salud que van desde las enfermedades profesionales adquiridas en el trabajo, los accidentes de todo tipo debido a un entorno crecientemente agresivo, la contaminación del aire, agua y medio ambiente hasta llegar a las enfermedades de la civilización, enfermedad yatrógenas y dolencias debidas específicamente a nuestra forma de vida presente. El hecho de que esta forma pueda ser patógena, en muchas ocasiones, no debe hacer olvidar que la misma ha posibilitado progresos extraordinarios en sanidad, lucha contra las enfermedades infecciosas y reducción de la mortalidad infantil. A pesar de todos los razonamientos esgrimidos contra nuestra forma de vida presente, son muy pocas las personas que escogen el abandonarla para regresar a formas de convivencia más primitivas. La evidencia prueba por otra parte que los estados de salud de los primitivos no se han acercado mucho al estado de bienestar total, ya que en la mayoría de estas sociedades las enfermedades han sido más abundantes y la esperanza de vida menor que en nuestras condiciones contemporáneas «patógenas». Solamente algunas sociedades como las de los esquimales o los isleños de la Polinesia poseían antes de entrar en contacto con el «hombre blanco» un grado aceptable de salud física y felicidad personal, pero, como señala Toynbee, ${ }^{14}$ los seres de estas sociedades se hallaban degradados por la limitación de sus actividades, pareciéndose poco al tipo de hombre global propuesto como modelo por los pensadores occidentales. Estas sociedades primitivas se habían detenido en su desarrollo y se parecían más a las sociedades de abejas u hormigas que estudia la etología que a las sociedades formadas por seres humanos como sujetos capaces de invención y cambio que estudia la sociología.

La concepción ideal de salud puede pues ser positiva si sirve para señalar constantemente nuevos objetivos para la práctica médica y sanitaria mejorando la situación existente en cada momento histórico. Pero la concepción ideal de salud puede ser negativa si lleva a prescindir de los innumerables logros médicos y sanitarios contemporáneos en aras a conseguir una salud utópica más propia de comunidades aisladas primitivas que de las condiciones cambiantes y dinámicas de casi todo el mundo contemporáneo.

Ricardo Moragas

Juan d'Orpí 16

Barcelona 17

14. Citado por Dubos en ibid, p. 224. 